Lukas Bier ${ }^{1}$, Philip Joisten ${ }^{1}$, Bettina Abendroth ${ }^{1}$

${ }^{1}$ Institut für Arbeitswissenschaft, Technische Universität Darmstadt

\title{
Warum nutzt der Mensch bevorzugt das Auto als Verkehrsmittel? Eine Analyse zum erlebten Fahrspaß unterschiedlicher Verkehrsmittelnutzer
}

\section{Schlüsselwörter}

Fahrspaß, Fahrerlebnis, Verkehrsmittelwahl

\section{Zusammenfassung}

Positives Fahrerleben ist ein zentraler Faktor, der die Wahl unserer Verkehrsmittel beeinflusst. Vor dem Hintergrund neuer, innovativer Möglichkeiten der Fortbewegung, wie dem automatisierten Fahren, stellt sich die Frage, was positives Fahrerleben ausmacht. Das Ziel dieses Beitrags ist zu bestimmen, warum der Mensch den Pkw als Verkehrsmittel bevorzugt und welche Faktoren bei der Nutzung verschiedener Verkehrsmittel zum Fahrspaß beitragen. Zur Klärung dieser Fragen wurden literaturbasiert Faktoren abgeleitet, die den Fahrspaß unterschiedlicher Verkehrsmittelnutzer prägen. Diese wurden anhand einer Befragungsstudie $\left(\mathrm{n}_{\text {ges }}=\right.$ 334) empirisch untersucht. Unterschiede hinsichtlich des Fahrspaßes von Verkehrsmittelnutzern wurden analysiert. Zusammenhangsanalysen legten Faktoren offen, die zum Fahrspaß von Verkehrsmittelnutzern beitragen. Die Analyse hinsichtlich des Fahrspaßes zeigt, dass sich die Einflussfaktoren in die Dimensionen Fahrer, Fahrzeug und Umwelt gliedern lassen. Einflüsse von soziodemografischen Merkmalen, sowie Merkmalen der Fahrt und den Eigenschaften des Pkws auf den Fahrspaß konnten identifiziert werden.

\section{Praktische Relevanz}

Die Beantwortung der Frage, was den Spaß beim Autorfahren im Vergleich zu der Nutzung anderer Verkehrsmittel ausmacht und die hier dargestellten Erkenntnisse können dazu beitragen, die Nutzung alternativer Verkehrsmittel attraktiver zu gestalten und so insbesondere in Gebieten mit starker Verkehrsdichte eine Entlastung der Straßen zu erreichen.

\section{Why do people prefer the car? Analysis regarding the driving enjoyment of different traffic participants}

\section{Keywords}

Driving enjoyment, driving experience, choice of transport mode

\section{Abstract}

Positive driving experience is a central factor regarding the choice of transport mode. Autonomous driving and other innovative means of transport place emphasis on the question which factors contribute to a positive driving experience. The aim of this research is to determine why people prefer the car as a mode of transport and to investigate which factors contribute to the driving enjoyment of users of different means of transport. Therefore, factors which influence the driving enjoyment of different means of transport were derived from literature and a survey was carried out $(n=334)$. 
Differences regarding the driving enjoyment of users of different means of transport were analyzed. Correlation analysis verified factors which influence the driving enjoyment of users of different means of transport. The analysis reveals that the factors of driving enjoyment can be grouped in the dimension driver, vehicle and environment. Influences of sociodemographic attributes as well as characteristics of the drive and properties of the car regarding the driving enjoyment could be identified.

\section{Practical Relevance}

The answer to the question of what makes driving a car fun compared to using other means of transport and the findings presented here can help to make the use of alternative means of transport more attractive and thus relieve the burden on roads, especially in areas with heavy traffic.

\section{Einleitung und Motivation}

Noch nie gab es so viel Mobilität und Verkehrsaufkommen wie heute. Moderne Verkehrsmittel sind schnell, kommen immer weiter, werden immer kostengünstiger und zeichnen sich durch eine stetig zunehmende Sicherheit aus (Merki 2008). Der Pkw ist heute das am häufigsten genutzte Verkehrsmittel in Deutschland (Eisenmann et al. 2018) und für viele zu einem unentbehrlichen Alltagsgegenstand geworden. Positives Fahrerleben beeinflusst die Wahl unserer Verkehrsmittel (Mokhtarian \& Salomon 2001; Steg 2005) und ist ein „zentraler Faktor für den Erfolg eines Automobils“ (Tischler \& Renner 2007, S. 105). Der Begriff Fahrspaß wird von Medien und Herstellern in diesem Zusammenhang häufig verwendet (Hagman 2010). Fahrspaß wird dabei von den Herstellern vor allem in Verbindung mit den fahrdynamischen Eigenschaften des Pkws gebracht. Auch in der Automobilfachpresse wird der Begriff Fahrspaß in Verbindung mit Geschwindigkeit, Fahrverhalten und -dynamik und Sportlichkeit beschrieben. Es bleibt unklar, was unter dem Begriff Fahrspaß aus wissenschaftlicher Sicht genau verstanden wird, und welche Faktoren den Fahrspaß beeinflussen.

Heute werden das Fahrerlebnis und der Fahrspaß insbesondere im Hinblick auf die immer weiter fortschreitende Automatisierung des Autofahrens diskutiert (vgl. Simon et al. 2015; Müller et al. 2017). Denn im Zuge der Automatisierung nimmt der Fahrer eine immer passivere Rolle ein. Folglich gleicht eine Autofahrt in Zukunft vielleicht mehr einer Bahnfahrt als einer Autofahrt im heutigen Sinne. Eine zentrale Fragestellung ist, wie sich automatisiertes Fahren auf den erlebten Fahrspaß auswirkt (Simon et al. 2015).

Das positive, emotionale Empfinden bzw. die Freude während der Nutzung eines Verkehrsmittels ist ein zentrales Motiv der Verkehrsmittelwahl (Ory \& Moktharian 2005). Die Motivationspsychologie bezeichnet dies als affektive Motive (Steg et al. 2001). Affektive Motive der Verkehrsmittelwahl begründen sich in positiven, emotionalen Empfindungen bzw. Spaß bei der Nutzung eines Verkehrsmittels und führen dazu, dass Individuen die Fahrt oder Nutzung eines Verkehrsmittels als solche schätzen. Sowohl eine intrinsische Motivation des Individuums ein bestimmtes Verkehrsmittel zu nutzen, als auch sekundäre Aktivitäten während der Fahrt, können positive, emotionale Gefühle bei einem Individuum auslösen und so zu dessen Fahrspaß beitragen (Mokhtarian \& Salomono 2001; Páez \& Whalen 2010). Empirische Arbeiten zu affektiven Motiven der Verkehrsmittelwahl zeigen, dass der Pkw als Verkehrsmittel in der Regel mit positiven Gefühlen assoziiert wird (Ellaway et al. 2003; Gatersleben \& Uzzell 2007; Gatersleben 2007; Kent 2014; Mann \& Abraham 2006; Steg 2003). Der Pkw unterscheidet sich von anderen Verkehrsmitteln in der 
besonderen Form der autonomen Mobilität (Automobilität). Autonomie ist also schon immer ein zentraler Aspekt der Pkw-Nutzung (Gatersleben 2007; Marsh \& Collett 1987), der häufig in Verbindung mit den Faktoren Kontrolle, Unabhängigkeit und/oder Freiheit genannt wird (Gatersleben 2007; Lupton 2002; Mann \& Abraham 2006; Steg 2003). Die Einschränkung der Autonomie bzw. Kontrolle oder Freiheiten des Individuums wirkt wiederum nachteilig und kann negative Gefühle produzieren. Gardner und Abraham (2007) zeigen, dass die Nutzung von öffentlichen Verkehrsmitteln mit einer Delegation von Kontrolle assoziiert wird, die bei Verkehrsmittelnutzern als ein Gefühl von Hilflosigkeit wahrgenommen wird.

Unklar ist bislang, welche Faktoren genau das Unabhängigkeitsgefühl verstärken, welche weiteren Faktoren zum Fahrspaß beitragen und ob Fahrspaß auch bei der Nutzung von weniger autonomen Verkehrsmittel aufkommen kann.

Das Ziel dieses Beitrags ist es somit zu bestimmen, welche Verkehrsmittel der Mensch bevorzugt und mit welchen Fortbewegungsmitteln Fahrspaß erreicht werden kann. Zudem sollen Faktoren identifiziert werden, die der Mensch bei der Nutzung unterschiedlicher Verkehrsmittel mit Fahrspaß verbindet.

Der Beitrag ist wie folgt aufgebaut: In Abschnitt 2 wird Fahrspaß in Abhängigkeit der Verkehrsmittelwahl modelliert. Abschnitt 3 beschreibt die Methodik der empirischen Studie. Abschnitt 4 gibt die Ergebnisse der durchgeführten Erhebung wieder. Abschnitt 5 und 6 beinhalten die Diskussion der Ergebnisse und das Fazit der Untersuchung.

\section{Modellierung des Fahrspaßes unterschiedlicher Verkehrsmittelnutzer}

Obwohl Fahrspaß ein sehr populärer Begriff ist, der häufig in der Automobilfachpresse und von Automobilherstellern verwendet wird, ist der Begriff bis heute nicht eindeutig definiert. Für diese Arbeit soll der Begriff Fahrspaß in Anlehnung an Tischler (2013, S. 148) verwendet werden: Fahrspaß ist ein positiver emotionaler Zustand einer Person, der durch ein momentan sinnliches Erleben der Fahrsituation bestimmt wird. In diesem Sinne ist Fahrspaß eine Komponente des gesamten Fahrerlebnisses, das die gesamte emotionale Reaktion eines Individuums auf die Fahrt mit einem Verkehrsmittel abbildet.

ebenso unklar ist, welche Faktoren auf den Fahrspaß unterschiedlicher Verkehrsmittelnutzer einwirken. Um diese Forschungslücke zu adressieren, soll in diesem Abschnitt ein Modell vorgestellt werden, dass als Grundlage für die folgenden Ausführungen dient (s. Bild 1). Das Modell beruht auf der Annahme, dass Fahrspaß aus dem Zusammenwirken von Faktoren resultiert, die den drei Dimensionen Fahrer, Fahrzeug und Umwelt zugeordnet werden können (Pöschel et al. 2010; Tischler \& Renner 2007). Des Weiteren nimmt die Fahraufgabe, wie beispielsweise der Wegezweck oder der Fahrtzeit, einen direkten Einfluss auf die Verkehrsmittelwahl (Mokhtarian et al. 2015) und wird deshalb in das Modell als vierte Dimension integriert.

Bild 1: Fahrspaß in Abhängigkeit der Verkehrsmittelwahl im System FahrerFahrzeug-Umwelt

Figure 1: Driving enjoyment in dependence of the choice of transport mode in the system driver-vehicle-environment

Bild 1 bitte hier einfügen

Entsprechend vorheriger Ausführungen sind die persönlichen Eigenschaften des Fahrers bzw. Nutzers von Verkehrsmitteln sind zentrale Faktoren, die die 
Verkehrsmittelwahl und den hieraus resultierenden Fahrspaß prägen. Neben soziodemografischen Merkmalen identifizieren Ory \& Mokhtarian (2005) Einstellung, Persönlichkeit und Lebensstil als entscheidende Einflussfaktoren für das Erleben von Fahrspaß. Die Charakteristiken des Individuums bestimmen den Fahrspaß maßgeblich. Als ausschlaggebend können hierbei soziodemografische Merkmale, wie das Alter und Geschlecht, sowie Einstellungen, Absichten, Gewohnheiten, der aktuelle Zustand eines Individuums und eventuell vorliegende Krankheiten oder Behinderungen angesehen werden. Neben allgemeinen Persönlichkeitseigenschaften des Individuums stellen Aktivitäten während der Fahrt einen weiteren Aspekt dar, der der Dimension des Fahrers bzw. Nutzers zugeordnet wird und einen Einfluss auf den Fahrspaß nimmt (Páez \& Whalen 2010). Des Weiteren wurde die Umsetzung des gewünschten Fahrstils als zentral für die positive emotionale Bewertung bei der Nutzung von Verkehrsmitteln identifiziert (Tischer \& Renner 2007) und folglich in das Modell aufgenommen.

Positives und auch negatives Fahr-Erleben wird auch durch das Fahrzeug bzw. Verkehrsmittel als solches beeinflusst. Aus Sicht der Kraftfahrzeugtechnik wird Fahrspaß vor allem durch die fahrdynamischen Gegebenheiten des Fahrzeugs beeinflusst (Schoeggl et al. 1998). Im Vordergrund steht dabei die exakte Umsetzung des Fahrwunsches durch das Fahrzeug (Tischler \& Renner 2007). Dies betrifft zum einen die exakte Reaktion auf Lenkeingaben, die dem Bereich der Querdynamik des Automobils zugeschrieben wird (Wedlin et al. 1992). Zum anderen werden darunter Aspekte der Längsdynamik gefasst, die sich auf die Beschleunigungsfähigkeit des Fahrzeuges und dessen Brems- und Traktionseigenschaften auf unterschiedlichen Belägen bezieht (Tischler 2013). Auch der vermittelte Komfort des Fahrzeuges wirkt sich auf das Erleben von Fahrspaß aus (Borriello 2016).

Fahrspaß ist, neben den Charakteristiken des Fahrzeuges und des Individuums bzw. Fahrers, vor allem von den zugrundeliegenden Umgebungsbedingungen (Dimension Umwelt) abhängig (Hagman 2010). Die Nutzung von Verkehrsmitteln ist abhängig von kulturellen, sozialen und physikalischen Umgebungsbedingungen (Carreira et al. 2014; Tischler 2013). Umgebungsbedingungen verändern insbesondere die Komplexität der Fahraufgabe, das heißt sie haben einen messbaren Einfluss auf das Verhalten des Fahrers (Fuller 2011) und wirken auf dessen emotionales Erleben ein. Wichtige Aspekte sind hierbei die Eigenschaften der Fahrbahn, Witterungsbedingungen, die Anwesenheit anderer Verkehrsteilnehmer, sowie die bauliche Umwelt abseits der Fahrbahn.

Ein zentraler Bestandteil des Modells (s. Bild 1) ist die Verkehrsmittelwahl eines Individuums, die den Fahrspaß beeinflusst Das Individuum kann zwischen der Nutzung eines Pkws oder alternativer Verkehrsmittel, wie öffentlichen Verkehrsmitteln, dem Fahrrad oder Motorrad wählen. Zusätzlich wird die Gruppe der Pkw-Nutzer in die Rolle Fahrer und Beifahrer unterschieden. Eine zentrale Annahme ist, dass sich der Fahrspaß zwischen den Gruppen der verschiedenen Verkehrsmittelnutzer unterscheidet.

\section{Methodik}

Zur Untersuchung der zugrundliegenden Fragestellungen dieses Beitrags wurde das exploratives Untersuchungsdesign einer Befragung gewählt. Ziel der empirischen Erhebung war es, Kontraste zwischen verschiedenen Verkehrsmittelnutzern und Faktoren, die den Fahrspaß unterschiedlicher Verkehrsmittelnutzer beeinflussen, zu identifizieren. Auf die Formulierung und Überprüfung von Hypothesen wurde verzichtet. 


\subsection{Konzeptionierung der Befragungsstudie}

Die Zielgruppe der Befragung waren Personen, die eine Pkw-Fahrerlaubnis erwerben können. Dies schließt zunächst alle Personen ein, die älter als 16 Jahre sind. Ausgeschlossen von der Befragung waren Personen, die ein Verkehrsmittel hauptberuflich nutzen, zum Beispiel Berufskraftfahrer, Taxifahrer, weil das Motiv des Einkommenserwerbs bei diesem Personenkreis im Vordergrund steht. Die onlineVersion des Fragebogens wurde mit der Software SosciSurvey erstellt (www. soscisurvey.de). Zur Akquise von Befragungsteilnehmern wurde u.a. auf die Panel SurveyCircle (www.surveycircle.com) und PollPool (www.poll-pool.com) zurückgegriffen. Parallel wurde eine Papierversion des Fragebogens erstellt und verteilt. Online Version und Papierversion des Fragebogens stimmen inhaltich vollkommen überein.

\subsection{Aufbau des Fragebogens}

Der Fragebogen ist wie folgt aufgebaut: Abschnitt 1 beinhaltet Angaben zu soziodemografischen Merkmalen der Befragungsteilnehmer. In Abschnitt 2 werden Informationen zur Nutzung des Pkws und alternativer Verkehrsmittel erfasst. Zwei Schlüsselfragen des Fragebogens sind die Fragen nach dem am häufigsten für alltägliche Fahrten genutzten Verkehrsmittel auf der einen Seite und dem hierfür bevorzugten Wunschverkehrsmittel auf der anderen Seite. Die Unterscheidung zwischen genutzten und bevorzugten Verkehrsmittel reflektiert, dass sich zwischen Wunschverkehrsmittel und tatsächlich genutzten Verkehrsmittel erhebliche Differenzen ergeben können. Diese Differenzen können beispielsweise auf wirtschaftlichen Gründen oder mangelnder Verfügbarkeit von Verkehrsmitteln basieren. Die Befragungsteilnehmer konnten bei diesen Fragestellungen zwischen den Verkehrsmitteln Pkw, öffentliche Verkehrsmittel, Fahrrad und Motorrad wählen. Zudem wurde das Verkehrsmittel Pkw in die drei Rollen Fahrer, Fahrer \& Beifahrer und nur Beifahrer unterteilt. Die Auswahl dieser Verkehrsmittel repräsentiert die am gesamten Verkehrsaufkommen am häufigsten genutzten Verkehrsmittel (vgl. Eisenmann et al. 2018). In Abschnitt 3 des Fragebogens wurde der erlebte Fahrspaß mit dem bevorzugten Verkehrsmittel auf einer 11-stufigen Ratingskala, von 0 (kein $\mathrm{Spa}$ ) bis 10 (viel Spaß), bewertet. Ebenfalls im dritten Abschnitt sollte der Befragte für alle übrigen von inm genutzten Verkehrsmittel die Aussage „Ich habe Spaß mit diesem Verkehrsmittel" auf einer 5 Stufigen Skala bewerten. Die Antwortmöglichkeiten lauteten von links nach rechts: trifft nicht zu, trifft eher nicht zu, teils-teils, trifft eher zu und trifft zu.

In einem vierten Abschnitt bewerteten die Befragungsteilnehmer den Einfluss von insgesamt 29 Faktoren auf den von Ihnen erlebten Fahrspaß (s. auch Tabelle 4). Die 29 Faktoren wurden aus der recherchierten Literatur zu Fahrspaß zusammengetragen und durch Faktoren aus Werbung und Fachmagazinen ergänzt. Die einzelnen Faktoren wurden im Fragebogen jeweils in einer Aussage positiv mit dem Fahrspaß in Verbindung gebracht. Die Teilnehmer der Studie sollten auf einer 5 Stufigen Skala (Trifft nicht zu, trifft eher nicht zu, teils-teils, trifft eher zu und trifft zu) ihren Grad der Zustimmung zur jeweiligen Aussage angeben.

\subsection{Statistische Auswertung}

Zur Auswertung der Daten wurden Kontraste zwischen Gruppen von Verkehrsmittelnutzern analysiert. Die Prüfung der Normalverteilung der Daten ist im Einzelfall mit dem Shapiro-Wilk-Test durchgeführt worden. Die Mehrzahl der Daten sind nicht normalverteilt. Deshalb wurde die Analyse der Kontraste mit dem nonparametrischen Kruskal-Wallis-Test mit der Untersuchung von paarweisen post-hoc- 
Kontrasten vorgenommen. Für den Sonderfall von nur zwei Vergleichsgruppen wurde auf den analogen Mann-Whitney-U Test zurückgegriffen. Zur Bestimmung der Relevanz von signifikanten Unterschieden wurde die Effektstärke dnach Cohen (1988) berechnet. Ein Wert größer oder gleich 0,5 wird als ein mittlerer Effekt und ein Wert größer oder gleich 0,8 als ein großer Effekt interpretiert (Cohen 1988). Im Unterschied zu den verwendeten nicht-parametrischen interferenzstatistischen Verfahren, basieren die Zusammenhangsanalysen bezüglich des Einflusses der 29 Faktoren auf den Fahrspaß der Befragungsteilnehmer auf dem parametrischen Produkt-MomentKorrelation-Koeffizienten $\left(r_{x y}\right)$. Die Auswertung der Daten erfolgte mit Hilfe des Programms IBM $\AA^{\circ}$ SPSS $\circledast$ Statistics in der Version 24.0.0.2.

\section{Ergebnisse}

Die Gesamtgröße der Stichprobe beträgt $n_{\text {total }}=348$ Befragte, mit $n_{1}=329$ online Befragten und $\mathrm{n}_{2}=19$ Befragten mit Papierversion. Der bereinigte Datensatz, nach Ausschluss von Probanden mit sehr schneller Bearbeitungszeit (Kennwert ,TIME_RSI $>2,0)$ und mit hohen Anteil an fehlenden Antworten (> 20\%), umfasst $n_{\text {ges }}=334$ ausgefüllte Fragebögen. Der Befragungszeitraum betrug, vom 30. Juni bis 16. Juli 2017, insgesamt 17 Tage.

\subsection{Deskriptive Beschreibung der Stichprobe}

Im Mittel über alle Befragten ergibt sich ein Alter von 36,9 Jahren, der Median beträgt 30 Jahre. Die Altersgruppen 31 - 50 Jahre und älter als 50 Jahre sind jeweils zu annähernd $25 \%$ vertreten. Der Anteil von Männern (53,6 \%) und Frauen (46,4 \%) ist nahezu gleichverteilt (s. Tabelle 1).

Tabelle 1: Alter, Geschlecht und genutzte Verkehrsmittel des Kollektivs $(n=334)$

Table 1: Age, gender and used means of transport in the sample $(n=334)$

\section{Tabelle 1 bitte hier einfügen}

Pkw-Nutzer, die den Pkw vorwiegend als Fahrer, als Fahrer und Beifahrer oder Beifahrer nutzen, stellen mit 50,3\% die größte Gruppe der Verkehrsmittelnutzer. Die kleinste Gruppe sind Motorradfahrer mit ca. $4 \%$ der gesamten Stichprobe. Öffentliche Verkehrsmittel werden von $28,1 \%$ und das Fahrrad von $17,7 \%$ der Befragten am häufigsten genutzt.

\subsection{Verkehrsmittelwahl und bevorzugtes Verkehrsmittel}

Eine zentrale Fragestellung betrifft die Präferenz für ein bestimmtes Verkehrsmittel. Hierzu gaben die Befragten zum einen an, welches Verkehrsmittel sie für ihre alläglichen Fahrten tatsächlich am häufigsten nutzen. Zum anderen wurde das von den Befragten bevorzugte Verkehrsmittel für diese Fahrten erfasst. Tabelle 2 zeigt den Zusammenhang zwischen genutzten und bevorzugten Verkehrsmittel. Das tatsächlich genutzte Verkehrsmittel ist hierbei auf der Abszisse, das bevorzugte Verkehrsmittel auf der Ordinate dargestellt. Bei der Betrachtung des Diagramms werden Wechselanteile zwischen genutztem und bevorzugtem Verkehrsmittel deutlich.

Nur 41,6\% der Befragten würden den Pkw als Verkehrsmittel bevorzugt nutzen. Tatsächlich aber wird der Pkw von insgesamt 50,3\% der Befragten genutzt. Öffentliche Verkehrsmittel werden nur von 19,8\% der Befragten bevorzugt, aber von 28,1\% tatsächlich genutzt. Der Anteil der Befragten, die das Fahrrad als Verkehrsmittel 
bevorzugt nutzen, beträgt $25,4 \%$. Diejenigen, die das Fahrrad tatsächlich nutzen, machen $17,7 \%$ aus. $13,2 \%$ der Befragten geben an, das Motorrad als Verkehrsmittel bevorzugt zu nutzen. Tatsächlich wird es aber nur von 3,9\% genutzt.

Tabelle 2: Tabellarische Gegenüberstellung von genutzten Verkehrsmittel und bevorzugten Wunschverkehrsmittel $(n=334)$

Table 2: Tabular comparison of used and preferred means of transport $(n=334)$

\section{Tabelle 2 bitte hier einfügen}

\subsection{Fahrspaß mit dem bevorzugten Verkehrsmittel}

Im Vergleich des angegebenen Fahrspaßes der Gruppen von Verkehrsmittelnutzern erzielen Pkw-Fahrer, Motorradfahrer und Fahrradfahrer die höchsten Werte (s. Bild 2). Die Mittelwerte des Fahrspaßes von Pkw-Beifahrern und Nutzern von öffentlichen Verkehrsmitteln sind am niedrigsten.

Bild 2: Fahrspaß mit dem bevorzugten Wunschverkehrsmittel [1=kein Spaß ... $11=$ viel Spaß]

Figure 2: Driving enjoyment with the preferred means of transport

\section{Bild 2 bitte hier einfügen}

Der Kruskal-Wallis-Test ergibt, dass die Unterschiede hinsichtlich der zentralen Tendenzen in den Gruppen der Verkehrsmittel signifikant sind (Chi-Quadrat $=69,202$, $\mathrm{df}=5, p \leq 0,001)$. Anschließend durchgeführte Post-hoc-Tests (paarweiser Vergleich nach Mann-Whitney-U mit Bonferroni-Korrektur) zeigen, dass die Gruppen sich signifikant im Einzelvergleich unterscheiden. Insbesondere unterscheiden sich die Gruppen „Pkw, als Fahrer", „Fahrradfahrer" und „Motorradfahrer" von den Gruppen „Pkw, als Beifahrer" und „öffentliche Verkehrsmittel“ (s. Tabelle 3).

Tabelle 3: Effektstärken und $p$-Werte für die paarweisen Einzelvergleiche zum Fahrspaß bei Nutzung des bevorzugten Wunschverkehrsmittels

Table 3: Size effects and p-values for the pairwise comparison of driving enjoyment of the preferred means of transport

\section{Tabelle 3 bitte hier einfügen}

\subsection{Fahrspaß nach Altersgruppe und Geschlecht}

Der Vergleich des angegebenen Fahrspaßes in Abhängigkeit des Alters der Befragungsteilnehmer zeigt keine signifikanten Unterschiede (Chi-Quadrat $=3,59$, df $=2, p=0,17)$. Bei der Betrachtung von Subgruppen von Verkehrsmittelteilnehmern zeigt sich, dass Pkw-Fahrer in der Altersgruppe von 31 bis 50 Jahren einen signifikant geringeren Fahrspaß haben als Jüngere oder Ältere (s. Bild 3). Eine effektstarke geschlechterspezifische Bewertung des Fahrspaßes konnte nicht festgestellt werden.

Bild 3: Fahrspaß nach Altersgruppen (a) aller Befragten und (b) mit dem Pkw-Fahrer als Wunschverkehrsmittel $[1=$ kein Spaß $\ldots 11=$ viel Spaß]

Figure 3: Driving enjoyment of age groups (a) of the total sample and (b) with the car (as driver) as preferred means of transport 


\subsection{Einflussfaktoren auf den Fahrspaß unterschiedlicher Verkehrsmittelnutzer}

Im Folgenden wird analysiert, welche der abgefragten Faktoren den Fahrspaß unterschiedlicher Verkehrsmittelnutzer beeinflussen. Dazu wird der Zusammenhang zwischen den 29 Fahrspaß-Faktoren, die der Befragung zugrunde liegen, und dem erlebten Fahrspaß mit dem bevorzugten Wunschverkehrsmittel bestimmt.

Die Ergebnisse der durchgeführten Korrelationsanalysen sind in Tabelle 4 aufgeführt. Die Gruppe der Pkw-Fahrer weist insgesamt 16 Faktoren auf, die signifikant $(p \leq 0,05)$ mit dem erlebten Fahrspaß korrelieren. Von den 16 signifikanten Korrelationskoeffizienten sind 11 Korrelationskoeffizienten größer als 0,3 und belegen somit einen mittleren Effekt nach Cohen (1988). Die höchsten Korrelationen ergeben sich zwischen dem Fahrspaß der PkW Fahrer und den Faktoren "langen Strecken“ ( $r_{x y}$ $=0,439, p \leq 0,01)$, „schnell fahren“ ( $\left.r_{x y}=0,429, p \leq 0,01\right)$ und "kurvige Strecken“ ( $r_{x y}=$ $0,382, p \leq 0,01)$.

Befragten, die den Pkw bevorzugt als Beifahrer nutzen, zeigen vier negative, starke Korrelationskoeffizienten: Der Fahrspaß korreliert hier stark negativ mit dem starken Beschleunigen $\left(r_{x y}=-0,745, p \leq 0,05\right)$, dem schnellen Fahren $\left(r_{x y}=-0,642, p \leq 0,05\right)$, dem Fahren mit einem leistungsstarken Fahrzeug $\left(r_{x y}=-0,699, p \leq 0,05\right)$ und der Aktivität des Surfens im Internet während der Fahrt ( $\left.r_{x y}=-0,707, p \leq 0,05\right)$.

Der Fahrspaß von Fahrradfahrern korreliert signifikant positiv mit dem Faktor "Entspannung" $\left(r_{x y}=0,322 p \leq 0,01\right)$. Weitere signifikante Korrelationen treten nicht auf.

Die Motorradfahrer weisen insgesamt drei Faktoren auf, die signifikant mit dem Fahrspaß korrelieren. Positiv korreliert das ruhige und vorausschauende Fahren mit dem Fahrspaß von Motorradfahrern $\left(r_{x y}=0,345, p \leq 0,05\right)$. Die Faktoren „Prestige“ ( $r x y$ $=-0,331, p \leq 0,05)$ und „Aktivität: Musik“ $\left(r_{x y}=-0,319, p \leq 0,05\right)$ korrelieren signifikant negativ mit dem erlebten Fahrspaß.

Alle Korrelationen zwischen Fahrspaß und Einflussfaktoren in den Subgruppen „Pkw als Fahrer und Beifahrer" und "öffentliche Verkehrsmittel" sind nicht signifikant.

Tabelle 4: Einflussfaktoren auf den Fahrspaß unterschiedlicher Verkehrsmittelnutzer Table 4: Impact factors on the driving enjoyment of users of different means of transport

\section{Tabelle 4 bitte hier einfügen}

\section{Diskussion}

Im Folgenden werden die Ergebnisse diskutiert. Dafür wird zunächst auf die Verkehrsmittelwahl eingegangen und im Anschluss auf den Einfluss der abgefragten Faktoren auf den Fahrspaß.

\subsection{Der Zusammenhang zwischen genutzten und bevorzugten Verkehrsmittel}


Eine zentrale Fragestellung, die dieser Arbeit zugrunde liegt, ist die Frage nach der Präferenz für ein bestimmtes Verkehrsmittel. Hierzu gaben die Befragten zum einen an, welches Verkehrsmittel sie für ihre alltäglichen Fahrten tatsächlich am häufigsten nutzen. Zur Auswahl standen hierbei das Verkehrsmittel Pkw, in den Rollen Fahrer oder Beifahrer und „Fahrer \& Beifahrer“, sowie öffentliche Verkehrsmittel, das Fahrrad und das Motorrad. Zum anderen wurde das von den Befragten bevorzugte Wunschverkehrsmittel erfasst, das sie für ihre alltäglichen Fahrten am häufigsten nutzen würden. Es zeigt sich, dass ein hoher Zusammenhang zwischen tatsächlich genutzten und bevorzugten Verkehrsmittel vorhanden ist.

$50 \%$ der Befragten und damit die größte Gruppe nutzt den Pkw als Verkehrsmittel. Das am zweithäufigste genutzte Verkehrsmittel sind öffentliche Verkehrsmittel (28\%), gefolgt vom Fahrrad (18\%) und dem Motorrad (4\%). Hätten die Befragten die freie Wahl des Verkehrsmittels, würden noch $42 \%$ den Pkw nutzen. Das Fahrrad würden $25 \%$ der Befragten nutzen, gefolgt von öffentlichen Verkehrsmitteln (20\%) und dem Motorrad (13\%). Die Unterschiede von benutzen zu bevorzugten Verkehrsmittel zeigen Hinweise auf Wechselanteile zwischen den Verkehrsmitteln auf. Diese fallen zwischen den Verkehrsmitteln unterschiedlich hoch aus und weisen in verschiedene Richtungen. Folgend wird dies für die einzelnen Verkehrsmittel eingehend diskutiert.

Die Anzahl der Befragten, die den Pkw vorwiegend als Fahrer nutzen, würden zu 19\% das Motorrad bevorzugt als Verkehrsmittel nutzen. Fasst man Pkws und Motorräder unter dem Begriff „motorisierter Individualverkehr" zusammen, zeigt sich, dass ein Großteil der Pkw-Nutzer auch ein vergleichbares Verkehrsmittel bevorzugen würde. Nur jeweils ca. $10 \%$ bevorzugen öffentliche Verkehrsmittel oder das Fahrrad und damit ein Verkehrsmittel, das sich vom Pkw grundsätzlich unterscheidet. Dieser Wechsel könnte auf spezifischen Nachteilen des Pkw-Fahrens beruhen, wie beispielsweise Staus, Parkplatzsuche oder hohe Kosten für die Unterhaltung eines Pkws.

Nutzer von öffentlichen Verkehrsmitteln weisen eine hohe Streuung in den bevorzugten Wunschverkehrsmitteln auf. Der Wert der Übereinstimmung von genutzten und bevorzugten Verkehrsmittel ist in dieser Gruppe mit 48,9\% am geringsten. Dies lässt darauf schließen, dass Nutzer von öffentlichen Verkehrsmitteln am wenigsten zufrieden mit ihrem Transportmittel sind und gerne auf andere Verkehrsmittel zurückgreifen würden. 33\% würden den Pkw als Verkehrsmittel bevorzugen, gefolgt vom Fahrrad mit ca. 15\%. Die Gründe für die Bevorzugung des Pkws und Fahrrads von Nutzern von öffentlichen Verkehrsmitteln könnten in den spezifischen Vorteilen des Pkws gegenüber von öffentlichen Verkehrsmitteln liegen, wie beispielsweise Flexibilität und freie Verfügbarkeit.

Fahrradfahrer sind mit ihrer Verkehrsmittelwahl sehr zufrieden. 88\% der Befragten geben an, das Fahrrad auch zu bevorzugen, ziehen also keinen Wechsel des Verkehrsmittels in Betracht. Aktive Verkehrsmittel werden mit einer hohen Zufriedenheit in Verbindung gebracht (Páez \& Whalen 2010). Die hohen Zustimmungswerte für das Fahrrad sind vor dem Hintergrund der Überpräsenz von vielen jungen Verkehrsteilnehmern in der Untersuchung zu sehen. Zum einen ist das Fahrrad nicht für alle Wegstrecken geeignet. Tatsächlich zeigen die erhobenen Daten, dass Fahrradfahrer durchschnittlich die geringste Wegstrecke zurücklegen. Zum anderen könnten die Ergebnisse dadurch verzerrt sein, dass Fahrradfahren ein sportliches positives Image zugeschrieben wird. Die Befragten könnten folglich so geantwortet haben, um ein positives Selbstbild zu vermitteln.

Die Gruppe der Befragten, die das Motorrad als Verkehrsmittel nutzen, bevorzugen dieses Verkehrsmittel auch (92,3\%). Hierbei kann auf eine starke Bindung zum 
Verkehrsmittel geschlossen werden, da das Motorrad häufig nicht nur als Verkehrsmittel, sondern als Freizeitgegenstand angesehen wird.

Der Pkw wird von der größten Gruppe der Befragten genutzt und auch bevorzugt. Berücksichtigt man die Sonderrollen von Motorrad (Freizeitgegenstand) und Fahrrad (Einschränkung der Reichweite/verzerrtes Selbstbild), kann aus den vorliegenden Daten geschlossen werden, dass der Pkw klar vor anderen Verkehrsmitteln, insbesondere vor öffentlichen Verkehrsmitteln bevorzugt wird. Ursächlich hierfür sind wahrscheinlich, neben instrumentalen bzw. rationalen Motiven für die Bevorzugung des Pkws, Emotionen, die mit der Nutzung eines Verkehrsmittels verknüpft werden (vgl. Anable \& Gatersleben 2005).

\subsection{Fahrspaß: Unterschiede zwischen Verkehrsmittelnutzern und Einflussfaktoren}

Die Bewertung von Fahrspaß bei der Nutzung eines Pkws, als Fahrer oder Beifahrer, von öffentlichen Verkehrsmitteln, des Fahrrads oder des Motorrads zeigt, dass sich signifikante Unterschiede zwischen drei Gruppen finden lassen. Den höchsten Fahrspaß haben die Motorradfahrer gefolgt von Pkw-Fahrern und Fahrradfahrern. Hier kommt zum Ausdruck, dass das Motorrad vorwiegend für Freizeitaktivitäten eingesetzt wird. Da der Zweck der Nutzung eines Verkehrsmittels einen Einfluss auf das Erlebnis der Fahrt selbst hat (Anable \& Gatersleben 2005), ist das Motorradfahren stark mit dem Erleben von Spaß beim Fahren verknüpft. Die gilt auch in abgeschwächter Form für Pkw-Fahrer und Fahrradfahrer.

Den geringsten Spaß zeigen Pkw-Beifahrer und Nutzer des ÖPV. Beide Gruppen können nicht aktiv auf das Verkehrsmittel einwirken, was mit einer Art von Kontrollverlust einhergeht (Gatersleben \& Uzzell 2007). Dieser Kontrollverlust ist bedingt durch die Rolle des Individuums als "hilfloser" Passagier und kann negative Emotionen auslösen (Gardner \& Abraham 2007). Deshalb steht hier der Zweck des befördert werden im Vordergrund.

Der Fahrspaß unterscheidet sich zwischen den Altersgruppen. Betrachtet man nur die Gruppe der Pkw-Fahrer, zeigt sich, dass Pkw-Fahrer in der Altersgruppe von 31 bis 50 Jahre einen geringeren Fahrspaß haben als Jüngere oder Ältere. Eine Erklärung hierfür kann sein, dass die mittlere Altersgruppe den Pkw vor allem für die Fahrt zur Arbeit nutzt und stärker durch Staus, zähflüssigen Verkehr und die Parkplatzsuche beeinträchtigt wird. Diese Umstände wurden als negativ für den Fahrspaß eingeschätzt. Jüngere Fahrer wie auch ältere haben eine geringere Fahrleistung und sind flexibel beim Einsatz ihres Pkws. Ein typisches Beispiel hierfür sind junge Erwachsene, die am Wochenende das Auto der Eltern nutzen oder das ältere Ehepaar, das ein Cabrio für eine Freizeitfahrt nutzt. Die Ergebnisse deuten darauf hin, dass die Beziehung zwischen Alter und Fahrspaß nichtlinear ist (vgl. auch Mokhtarian et al. 2015).

In den Ergebnissen finden sich allenfalls tendenzielle, geschlechtsspezifische Unterschiede für den Fahrspaß. Die Ansicht, dass Männer mehr Fahrspaß haben, da sie den Pkw häufiger nutzen und ein tiefergehendes Interesse am Pkw selbst haben (Ellaway et al. 2003), ist in dieser Untersuchung nicht signifikant zu belegen.

Insgesamt wurde der Einfluss von 29 Faktoren auf den Fahrspaß von Verkehrsmittelnutzern abgefragt. Pkw-Fahrer zeigen die höchsten signifikanten und relevanten Korrelationen zum Fahrspaß. Die übrigen Gruppen weisen nur vereinzelt Korrelationen auf. Dementsprechend wird der Fahrspaß von Pkw-Fahrern durch die Items des Fragebogens am besten erfasst. 
Die höchste Korrelation weisen Pkw-Fahrer zu dem Faktor „lange Strecken“ auf. Dieser Zusammenhang lässt darauf schließen, dass den Personen mit hohem Fahrspaß eine intrinsische Motivation zugrunde liegt: Sie verbinden die Fahrt von längeren Strecken mit einem höheren Spaß (Mokhtarian \& Salomon 2001). Eine weitere Erklärung für diesen Zusammenhang könnte im Ziel der Fahrt liegen. Längere Fahrten sind oftmals mit Freizeitaktivitäten bzw. der Fahrt in den Urlaub verbunden. Die Befragten könnten positive Emotionen, die während der Fahrt mit dem Verkehrsmittel selbst entstehen mit positiven Emotionen vermischen, die aus dem Fahrtziel resultieren (Mokhtarian et al. 2015).

Weitere Faktoren, die hoch mit dem Fahrspaß von Pkw-Fahrer korrelieren sind „schnell fahren“, „starke Beschleunigung“, „Fahren im Gelände“ und „leistungsstarkes Fahrzeug". Es zeigt sich, dass der Fahrspaß von Pkw-Fahrern durch aktives, sportliches Fahren entsteht. Entspanntes Fahren ist hingegen für den Fahrspaß von Pkw-Fahrern nicht relevant. Dies bestätigt Erkenntnisse aus der Literatur, die Fahrspaß mit aktiven, sportlichen Fahren umschreiben (Engelbrecht 2013). Nichtdestotrotz ist nicht allein das Fahrzeug für den Fahrspaß verantwortlich, sondern auch die befahrene Strecke und das Wetter spielen eine wichtige Rolle.

Die Gruppe der Pkw-Beifahrer weisen stark negative Korrelationen zu den Faktoren "leistungsstarkes Fahrzeug“, „starke Beschleunigung“ und „schnell fahren“ auf. Die Faktoren, die den Fahrspaß von Pkw-Beifahrern ausmachen sind folglich konträr zu den Faktoren der Pkw-Fahrer. Die Fahrsituation (Pkw-Fahrer vs. Pkw-Beifahrer) nimmt also einen erheblichen Einfluss auf die Faktoren, die Fahrspaß bedingen. Dieses Ergebnis muss jedoch vor dem Hintergrund der kleinen Stichprobe der Pkw-Beifahrer $(n=10)$ eingeordnet werden, jedoch weist dieser Befund darauf hin, dass der Fahrspaß beim passiven Beifahren durch andere Faktoren geprägt ist als beim aktiven Fahren.

Ein weiteres Ergebnis ist, dass sekundäre Aktivitäten während der Fahrt keinen Einfluss auf den Fahrspaß nehmen. Es wurde angenommen, dass sekundäre Aktivitäten zu einem positiven Nutzen der Fahrt beitragen (Páez \& Whalen 2010). Diese Annahme konnte in dieser Untersuchung nicht bestätigt werden. Lediglich das Führen von Gesprächen steht mit dem Fahrspaß von Pkw-Fahrern in einem positiven Zusammenhang. Dies weist darauf hin, dass soziale Interaktion während der Fahrt vom Pkw-Fahrer als positiv wahrgenommen wird (Mokhtarian et al. 2015).

\section{Fazit}

Fahrspaß ist eine Komponente des Fahrerlebnisses, das die gesamte emotionale Reaktion eines Individuums auf das Erleben aller Faktoren der Interaktion MenschFahrzeug-Umwelt umfasst. In dieser Untersuchung konnten eine Reihe von Faktoren, die den Fahrspaß und das Fahrerlebnis beeinflussen, explorativ bestätigt werden. Der Einfluss verschiedener anderer Faktoren konnte hingegen nicht bestätigt werden. Die Modellannahme, dass sich die Faktoren des Fahrspaßes in die Dimensionen Fahrer, Fahrzeug, Umwelt und Fahraufgabe gliedern lässt, kann bestätigt werden. Es zeigt sich, dass sich der Fahrspaß von aktiven und passiven Verkehrsmitteln grundlegend unterscheidet. Während Pkw-Fahrer Fahrspaß am schnellen, aktiven Fahren haben, wirkt sich dies negativ auf den Fahrspaß von Pkw-Beifahrern aus. Im Zuge einer Automatisierung des Fahrzeuges muss diesem Umstand besondere Bedeutung zugesprochen werden, da Fahrer zukünftig zu einer Art Beifahrer im eigenen Fahrzeug werden. Die Ergebnisse legen die Vermutung nahe, dass Fahrspaß bei automatisierten Fahrzeugen nicht durch starke Motorleistung und schnelles Fahren erzielt werden kann. 
Auch konnte der Einfluss von soziodemografischen Merkmalen auf den Fahrspaß bestätigt werden. Weitere Faktoren, die (tendenziell) auf den Fahrspaß Einfluss nehmen, konnten in den Merkmalen der Fahrt und den Eigenschaften des Pkws identifiziert werden.

Instrumentale und affektive Motive sind ausschlaggebend dafür, dass der Mensch den Pkw als Verkehrsmittel bevorzugt. Ausschlaggebend ist, dass der Pkw als bequem, schnell und wirtschaftlich angesehen wird. Gefühle von Autonomie, Freiheit und Kontrolle über viele Aspekte der Fahrt führen zu positiven Gefühlen, die sich in der Präferenz des Individuums für den Pkw äußern.

Fahrspaß ist multidimensional und von intrinsischen Prozessen bestimmt, die auf externe Reize reagieren. Es ist weitere Forschungsarbeit nötig, um die Entstehung von Fahrspaß und Einflussfaktoren auf diesen zu erfassen.

\section{Literatur}

Anable J, Gatersleben B (2005) All work and no play? The role of instrumental and affective factors in work and leisure journeys by different travel modes. Transport Res A-Pol 39:163-181

Borriello A (2016) Pleasure of driving components: a partial least square - path modeling appraoch. 16th Swiss Transportation Research Conference, Monte Verità / Ascona

Carreira R, Patrício L, Natal Jorge R, Magee C (2014) Understanding the travel experience and its impact on attitudes, emotions and loyalty towards the transportation provider-A quantitative study with mid-distance bus trips. Transp Policy 31:35-46

Cohen J (1988) Statistical Power Analysis for the Behavioral Sciences. 2. Aufl., Academic Press, New York

Eisenmann C, Chlond B, Hilgert, T, von Behren S, Vortisch P (2018) Deutsches Mobilitätspanel (MOP). Wissenschaftliche Begleitung und Auswertung. Bericht 2016/2017: Alltagsmobilität und Fahrleistung (Institut für Verkehrswesen, Hrsg.). Karlsruhe: Karlsruher Institut für Technologie (KIT)

Ellaway A, Macintyre S, Hiscock R, Kearns A (2003) In the driving seat. Psychosocial benefits from private motor vehicle transport compared to public transport. Transport Res F-Traf 6:217-231

Engelbrecht A (2013). Fahrkomfort und Fahrspaß bei Einsatz von Fahrerassistenzsystemen. Dissertation, Humboldt-Universität zu Berlin

Fuller R (2011) Driver control theory. From task difficulty homeostasis to risk allostasis. In B E Porter (Hrsg.) Handbook of traffic psychology. Elsevier Science, Amsterdam, 13-26

Gardner B, Abraham C (2007) What drives car use? A grounded theory analysis of commuters' reasons for driving. Transport Res F-Traf 10:187-200

Gatersleben B, Uzzell D (2007) Affective Appraisals of the Daily Commute. Comparing Perceptions of Drivers, Cyclists, Walkers, and Users of Public Transport. Environ Behav 39:416-431 
Gatersleben B (2007) Affective and Symbolic Aspects of Car Use. In L Steg, T Garling (Hrsg.) Threats from Car Traffic to the Quality of Urban Life. Problems, Causes, Solutions. Bingley: Emerald Group Publishing Limited. 219-233

Hagman O (2010) Driving Pleasure. A Key Concept in Swedish Car Culture. Mobilities 5:25-39

Jakobsson C (2007) Instrumental Movites for Private Car Use. In L Steg, T Garling (Hrsg.) Threats from Car Traffic to the Quality of Urban Life. Problems, Causes, Solutions. Bingley: Emerald Group Publishing Limited, 205-217

Kent J L (2014) Driving to save time or saving time to drive? The enduring appeal of the private car. Transport Res A-Pol 65:103-115

Lupton D (2002) Road rage: drivers' understandings and experiences. J Sociol 38:275-290

Mann E, Abraham C (2006) The role of affect in UK commuters' travel mode choices. An interpretative phenomenological analysis. Brit J Psychol 97:155-176

Marsh P E, Collett P (1987) Driving passion. The psychology of the car. Faber and Faber, Boston

Merki C M (2008) Verkehrsgeschichte und Mobilität. Ulmer-Verlag, Stuttgart

Mokhtarian P L, Papon F, Goulard M, Diana M (2015) What makes travel pleasant and/or tiring? An investigation based on the French National Travel Survey. Transportation 42:1103-1128

Mokhtarian P L, Salomon I (2001) How derived is the demand for travel? Some conceptual and measurement considerations. Transport Res A-Policy 35:695-719

Müller A, Stockinger C, Walter J, Heuser T, Abendroth B, Bruder R. (2017) Einflussfaktoren auf die Akzeptanz des automatisierten Fahrens aus der Sicht von Fahrerinnen und Fahrer. 8. Darmstädter Kolloquium „mensch + fahrzeug“

Ory D T, Mokhtarian P L (2005) When is getting there half the fun? Modeling the liking for travel. Transport Res A-Pol 39:97-123

Páez A, Whalen K (2010) Enjoyment of commute. A comparison of different transportation modes. Transport Res A-Pol 44:537-549

Pöschel K, Kienast H, Spanner-Ulmer B (2010) Entwicklung und Evaluation eines Fragebogens zur Messung von Fahrspaß. VDI-Verlag, Düsseldorf

Schoeggl P, List H O, Fraidl G K, Hischmann A (1998) Echtzeitsimulation des subjektiven Fahrbarkeitsempfinden. VDI-Verlag: Düsseldorf

Simon K, Jentsch M, Bullinger A C, Schamber G, Meincke E. (2015) Sicher aber langweilig? Auswirkungen vollautomatisierten Fahrens auf den erlebten Fahrspaß. Z Arb Wiss 69:81-88

Steg L (2003) Can public transport compete with the private car? IATSS Research 27:27-35

Steg $L$ (2005) Car use: Lust and must. Instrumental, symbolic and affective motives for car use. Transport Res A-Pol 39:147-162.

Steg L, Vlek C, Slotegraaf G (2001) Instrumental-reasoned and symbolic-affective motives for using a motor car. Transport Res F-Traf 4:151-169 
Tischler M A (2013) Entwicklungsziel Fahrspaß. Gestaltungsempfehlungen für die Auslegung von Kraftfahrzeugen zur Optimierung des subjektiven Erlebens der Fahrzeugführung. Dissertation, Humboldt-Universität zu Berlin

Tischler M A, Renner G (2007) Ansatz zur Messung von positivem Fahrerleben. Die Messung von Fahrspaß und Ableitungen für die Fahrzeuggestaltung. VDI-Verlag, Düsseldorf

Wedlin J, Tillback L-R, Bane O (1992) Combining properties for driving pleasure and driving safety: A challenge for the chassis engineer. SAE SPEC PUBL 925:79-96

Anschrift der Verfasser
M.Sc. Lukas Bier
M.Sc. Philip Joisten
Dr.-Ing. Bettina Abendroth
Institut für Arbeitswissenschaft
Technische Universität Darmstadt
Otto-Berndt-Straße 2
D-64287 Darmstadt
E-Mail: I.bier@iad.tu-darmstadt.de

This is a post-peer-review, pre-copyedit version of an article published in "Zeitschrift für Arbeitswissenschaft". The final authenticated version is available online at: http://dx.doi.org/10.1007/s41449-018-00144-9 


\section{Bild 1}

Bild 1: Fahrspaß in Abhängigkeit der Verkehrsmittelwahl im System FahrerFahrzeug-Umwelt

Figure 1: Driving enjoyment in dependence of the choice of transport mode in the system driver-vehicle-environment

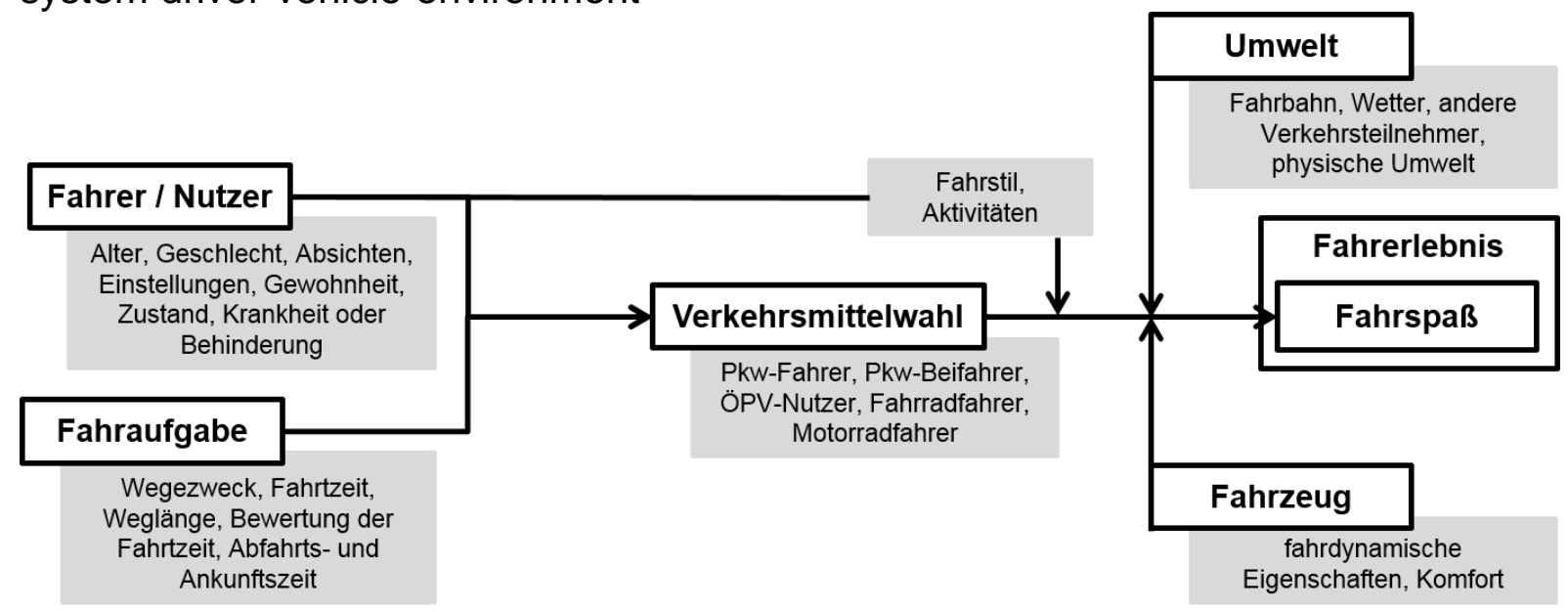




\section{Bild 2}

Bild 2: Fahrspaß mit dem bevorzugten Wunschverkehrsmittel [1=kein Spaß ... $11=$ viel Spaß]

Figure 2: Driving enjoyment with the preferred means of transport

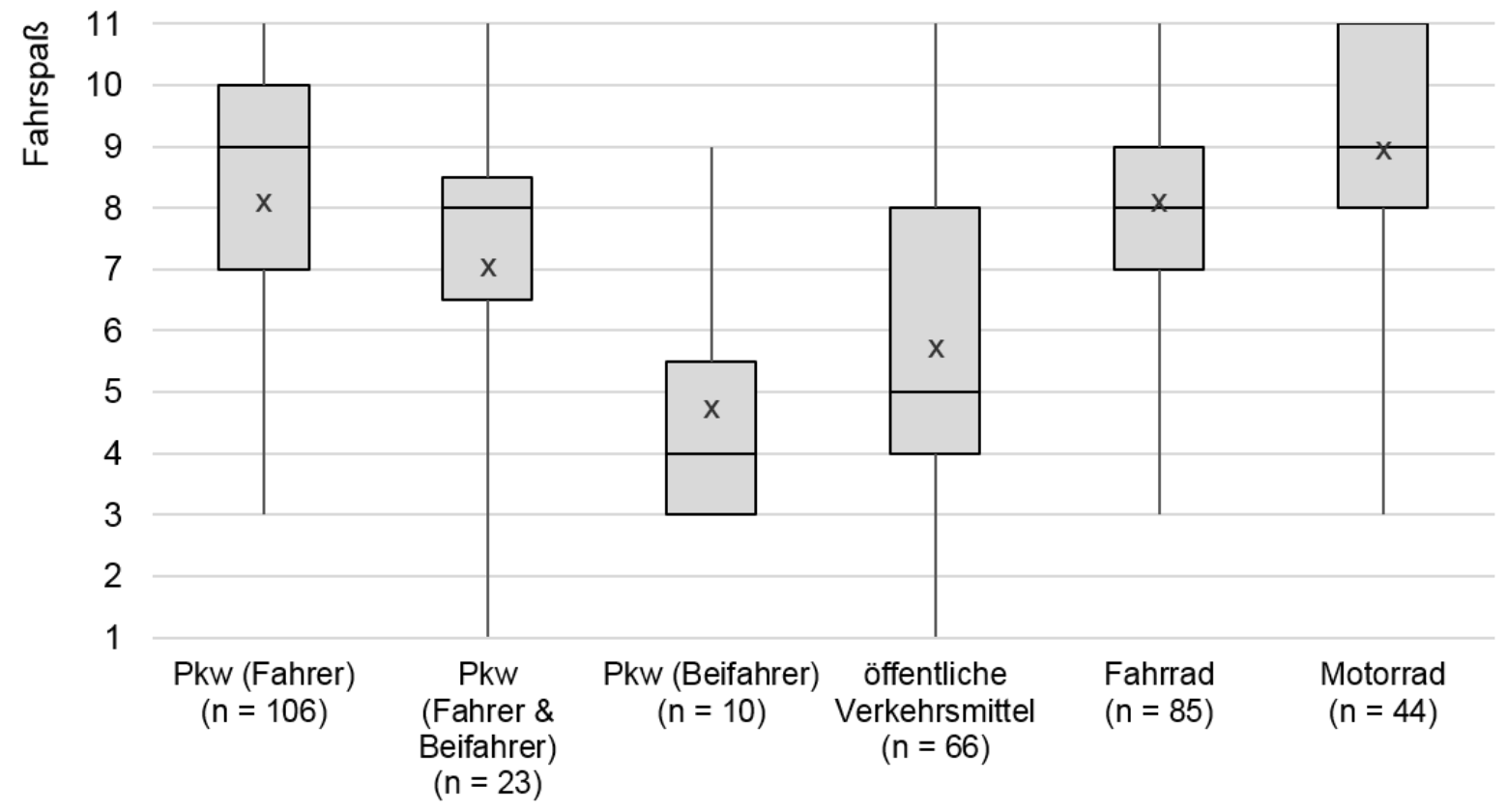




\section{Bild 3}

Bild 3: Fahrspaß nach Altersgruppen (a) aller Befragten und (b) mit dem Pkw-Fahrer als Wunschverkehrsmittel $[1=$ kein Spaß ... 11=viel Spaß]

Figure 3: Driving enjoyment of age groups (a) of the total sample and (b) with the car (as driver) as preferred means of transport

(a)

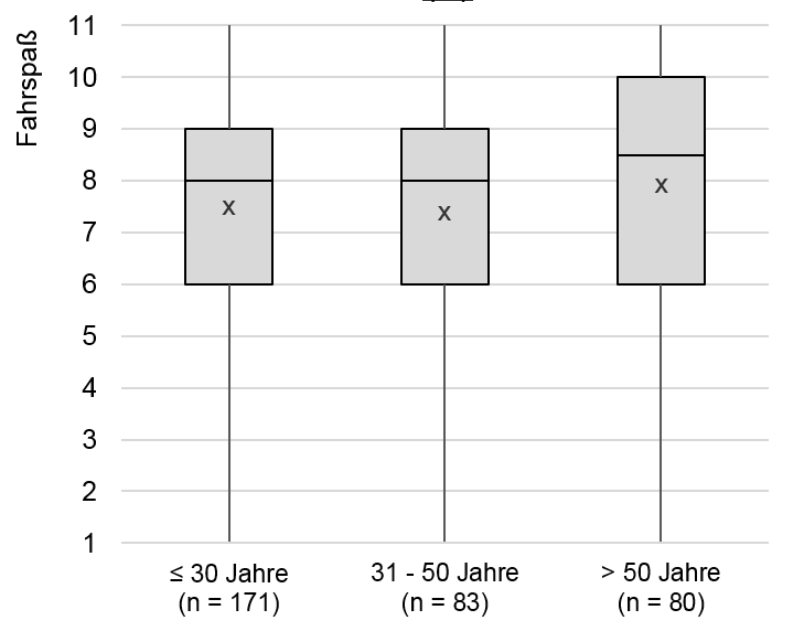

(b)

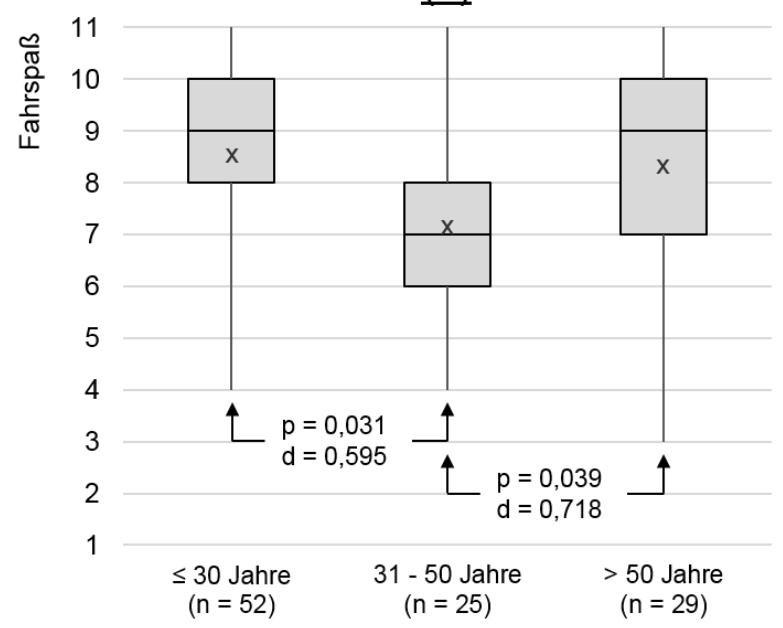


Tabelle 1

Tabelle 1: Alter, Geschlecht und genutzte Verkehrsmittel des Kollektivs ( $n=334)$ Table 1: Age, gender and used means of transport in the sample $(n=334)$

\begin{tabular}{|c|c|c|c|}
\hline \multirow{4}{*}{ Alter } & & $\begin{array}{c}\text { abs. } \\
\text { Anzahl }\end{array}$ & $\begin{array}{l}\text { rel. Anzahl } \\
(\%)\end{array}$ \\
\hline & $\leq 30$ Jahre & 171 & 51,2 \\
\hline & 31 - 50 Jahre & 83 & 24,8 \\
\hline & > 50 Jahre & 80 & 24,0 \\
\hline \multirow[t]{2}{*}{ Geschlecht } & weiblich & 155 & 46,4 \\
\hline & männlich & 179 & 53,6 \\
\hline \multirow{6}{*}{$\begin{array}{l}\text { Genutztes } \\
\text { Verkehrs- } \\
\text { mittel }\end{array}$} & Pkw, vorwiegend als Fahrer & 152 & 45,5 \\
\hline & $\begin{array}{l}\text { Pkw, vorw. Als Fahrer \& } \\
\text { Beifahrer }\end{array}$ & 14 & 4,2 \\
\hline & $\begin{array}{l}\text { Pkw, vorwiegend als } \\
\text { Beifahrer }\end{array}$ & 2 & 0,6 \\
\hline & Öffentliche Verkehrsmittel & 94 & 28,1 \\
\hline & Fahrrad & 59 & 17,7 \\
\hline & Motorrad & 13 & 3,9 \\
\hline
\end{tabular}




\section{$\underline{\text { Tabelle } 2}$}

Tabelle 2: Tabellarische Gegenüberstellung von genutzten Verkehrsmittel und bevorzugten Wunschverkehrsmittel $(n=334)$

Table 2: Tabular comparison of used and preferred means of transport $(n=334)$

Bevorzugtes Wunschverkehrsmittel

\begin{tabular}{|c|c|c|c|c|c|c|c|}
\hline $\begin{array}{l}\text { genutztes } \\
\text { Verkehrsmittel }\end{array}$ & $\begin{array}{c}\text { Pkw, als } \\
\text { Fahrer }\end{array}$ & $\begin{array}{l}\text { Pkw, als } \\
\text { Fahrer \& } \\
\text { Beifahrer }\end{array}$ & $\begin{array}{l}\text { Pkw, als } \\
\text { Beifahrer }\end{array}$ & $\begin{array}{c}\text { öffentliche } \\
\text { Verkehrs- } \\
\text { mittel }\end{array}$ & Fahrrad & $\begin{array}{l}\text { Motor- } \\
\text { rad }\end{array}$ & $\Sigma$ \\
\hline $\begin{array}{c}\text { Pkw, } \\
\text { vorwiegend als } \\
\text { Fahrer }\end{array}$ & $\begin{array}{c}84 \\
(55,3 \%)\end{array}$ & $\begin{array}{c}6 \\
(3,9 \%)\end{array}$ & $\begin{array}{c}2 \\
(1,3 \%)\end{array}$ & $\begin{array}{c}15 \\
(9,9 \%)\end{array}$ & $\begin{array}{c}16 \\
(10,5 \%)\end{array}$ & $\begin{array}{c}29 \\
(19,1 \%)\end{array}$ & $\begin{array}{c}152 \\
(100 \%)\end{array}$ \\
\hline $\begin{array}{l}\text { Pkw, vorw. als } \\
\text { Fahrer \& } \\
\text { Beifahrer }\end{array}$ & $\begin{array}{c}1 \\
(7,1 \%)\end{array}$ & $\begin{array}{c}7 \\
(50,0 \%)\end{array}$ & $\begin{array}{c}1 \\
(7,1 \%)\end{array}$ & $\begin{array}{c}2 \\
(14,3 \%)\end{array}$ & $\begin{array}{c}3 \\
(21,5 \%)\end{array}$ & & $\begin{array}{c}14 \\
(100 \%)\end{array}$ \\
\hline $\begin{array}{l}\text { Pkw, } \\
\text { vorwiegend als } \\
\text { Beifahrer }\end{array}$ & $\begin{array}{c}1 \\
(50,0 \%)\end{array}$ & & $\begin{array}{c}1 \\
(50,0 \%)\end{array}$ & & & & $\begin{array}{c}2 \\
(100 \%)\end{array}$ \\
\hline $\begin{array}{c}\text { öffentliche } \\
\text { Verkehrsmittel }\end{array}$ & $\begin{array}{c}17 \\
(18,1 \%)\end{array}$ & $\begin{array}{c}9 \\
(9,6 \%)\end{array}$ & $\begin{array}{c}5 \\
(5,3 \%)\end{array}$ & $\begin{array}{c}46 \\
(48,9 \%)\end{array}$ & $\begin{array}{c}14 \\
(14,9 \%)\end{array}$ & $\begin{array}{c}3 \\
(3,2 \%)\end{array}$ & $\begin{array}{c}94 \\
(100 \%)\end{array}$ \\
\hline Fahrrad & $\begin{array}{c}3 \\
(5,1 \%)\end{array}$ & $\begin{array}{c}1 \\
(1,7 \%)\end{array}$ & $\begin{array}{c}1 \\
(1,7 \%)\end{array}$ & $\begin{array}{c}2 \\
(3,4 \%)\end{array}$ & $\begin{array}{c}52 \\
(88,1 \%)\end{array}$ & & $\begin{array}{c}59 \\
(100 \%)\end{array}$ \\
\hline Motorrad & & & & $\begin{array}{c}1 \\
(7,7 \%) \\
\end{array}$ & & $\begin{array}{c}12 \\
(92,3 \%) \\
\end{array}$ & $\begin{array}{c}13 \\
(100 \%) \\
\end{array}$ \\
\hline$\Sigma$ & $\begin{array}{c}106 \\
(31,7 \%)\end{array}$ & $\begin{array}{c}23 \\
(6,9 \%)\end{array}$ & $\begin{array}{c}10 \\
(3,0 \%)\end{array}$ & $\begin{array}{c}66 \\
(19,8 \%)\end{array}$ & $\begin{array}{c}85 \\
(25,4 \%)\end{array}$ & $\begin{array}{c}44 \\
(13,2 \%)\end{array}$ & $\begin{array}{c}334 \\
(100 \%)\end{array}$ \\
\hline
\end{tabular}

Anmerkung: Die Prozentangaben beziehen sich auf die Zeilensumme 


\section{Tabelle 3}

Tabelle 3: Effektstärken und p-Werte für paarweise Einzelvergleiche zum Fahrspaß des bevorzugten Wunschverkehrsmittels

Table 3: Size effects and p-values for the pairwise comparison of driving enjoyment of the preferred means of transport

\begin{tabular}{|c|c|c|c|c|c|c|}
\hline Vergleich & $\begin{array}{l}\text { Pkw, als } \\
\text { Fahrer }\end{array}$ & $\begin{array}{l}\text { Pkw, als } \\
\text { Fahrer \& } \\
\text { Beifahrer }\end{array}$ & $\begin{array}{l}\text { Pkw, als } \\
\text { Beifahrer }\end{array}$ & $\begin{array}{c}\text { Öffentliche } \\
\text { Verkehrs- } \\
\text { mittel }\end{array}$ & Fahrrad & Motorrad \\
\hline Pkw, als Fahrer & - & n.s. & $\begin{array}{c}U=-124,2^{* *} \\
d=0,79\end{array}$ & $\begin{array}{c}U=-91,8^{\star \star} \\
d=1,06\end{array}$ & n.s. & n.s. \\
\hline $\begin{array}{l}\text { Pkw, als Fahrer \& } \\
\text { Beifahrer }\end{array}$ & n.s. & - & n.s. & n.s. & n.s. & $\begin{array}{l}U=-76,7^{*} \\
d=0,83\end{array}$ \\
\hline $\begin{array}{l}\text { Pkw, als } \\
\text { Beifahrer }\end{array}$ & $\begin{array}{c}U=124,2^{* *} \\
d=0,79\end{array}$ & n.s. & - & n.s. & $\begin{array}{c}U=-117,76^{*} \\
d=0,82\end{array}$ & $\begin{array}{c}U=-154,7^{* *} \\
d=1,62\end{array}$ \\
\hline $\begin{array}{l}\text { öffentliche } \\
\text { Verkehrsmittel }\end{array}$ & $\begin{array}{l}U=91,8^{\star *} \\
d=1,06\end{array}$ & n.s. & n.s. & - & $\begin{array}{c}U=-85,3^{\star *} \\
d=0,99\end{array}$ & $\begin{array}{c}U=-122,2^{* *} \\
d=1,42\end{array}$ \\
\hline Fahrrad & n.s. & n.s. & $\begin{array}{c}U=117,76^{*} \\
d=0,82\end{array}$ & $\begin{array}{l}U=85,3^{* *} \\
d=0,99\end{array}$ & - & n.s. \\
\hline Motorrad & n.s. & $\begin{array}{l}U=76,7^{*} \\
d=0,83\end{array}$ & $\begin{array}{c}U=154,7^{\star *} \\
d=1,62\end{array}$ & $\begin{array}{c}U=122,2^{* *} \\
d=1,42\end{array}$ & n.s. & - \\
\hline n.s. $=$ nicht signifikant & $* \mathrm{p}<0,05$ & $\mathrm{p}<0,001$ & & & & \\
\hline
\end{tabular}




\section{$\underline{\text { Tabelle } 4}$}

Tabelle 4: Einflussfaktoren auf den Fahrspaß unterschiedlicher Verkehrsmittelnutzer Table 4: Impact factors on the driving enjoyment of users of different means of transport

\begin{tabular}{|c|c|c|c|c|c|c|}
\hline & $\begin{array}{l}\text { Pkw, als } \\
\text { Fahrer }\end{array}$ & $\begin{array}{l}\text { Pkw, als } \\
\text { Fahrer \& } \\
\text { Beifahrer }\end{array}$ & $\begin{array}{l}\text { Pkw, als } \\
\text { Beifahrer }\end{array}$ & $\begin{array}{c}\text { öffentliche } \\
\text { Verkehrs- } \\
\text { mittel }\end{array}$ & Fahrrad & Motorrac \\
\hline$n$ & 106 & 23 & 10 & 66 & 85 & 44 \\
\hline Faktor & \multicolumn{6}{|c|}{ Produkt-Moment-Korrelation $r_{x y}$ zum Fahrspaß } \\
\hline freie Fahrt & $0,345^{\star *}$ & 0,107 & $-0,429$ & 0,165 & 0,065 & 0,198 \\
\hline Landschaft & 0,083 & $-0,197$ & 0,151 & 0,119 & 0,188 & $-0,048$ \\
\hline $\begin{array}{l}\text { zügig \& gleichmäßig } \\
\text { fahren }\end{array}$ & 0,174 & 0,000 & 0,213 & 0,092 & 0,029 & 0,094 \\
\hline Flow-Erlebnis & $0,284^{\star \star}$ & 0,279 & 0,271 & 0,132 & 0,136 & 0,144 \\
\hline Regen & 0,103 & $-0,235$ & 0,478 & 0,129 & 0,088 & $-0,095$ \\
\hline $\begin{array}{l}\text { kein langsamer Fahrer } \\
\text { voraus }\end{array}$ & $0,248^{\star}$ & 0,186 & $-0,345$ & 0,138 & 0,056 & 0,140 \\
\hline Aktivität: lesen & 0,005 & $-0,252$ & $-0,288$ & 0,082 & 0,133 & 0,079 \\
\hline kurze Strecken & $-0,008$ & $-0,130$ & $-0,240$ & 0,212 & 0,081 & $-0,155$ \\
\hline keine Parkplatzsuche & $0,205^{\star}$ & $-0,331$ & $-0,623$ & 0,108 & 0,122 & 0,157 \\
\hline leistungsstarkes Fahrzeug & $0,302^{\star \star}$ & 0,359 & $-0,699^{\star}$ & $-0,032$ & $-0,107$ & 0,008 \\
\hline starke Beschleunigung & $0,348^{* *}$ & 0,338 & $-0,745^{\star}$ & 0,060 & $-0,040$ & $-0,006$ \\
\hline Aktivität: Internet & 0,042 & $-0,388$ & $-0,707^{\star}$ & 0,011 & 0,108 & $-0,125$ \\
\hline Fahren im Gelände & $0,360^{\star *}$ & 0,279 & $-0,308$ & 0,030 & 0,208 & 0,002 \\
\hline Aktivität: Musik & 0,088 & $-0,072$ & $-0,301$ & 0,167 & $-0,092$ & $-0,319^{*}$ \\
\hline guter Straßenkontakt & $0,212^{*}$ & 0,058 & $-0,190$ & 0,134 & $-0,082$ & 0,254 \\
\hline Entspannung & 0,076 & $-0,181$ & $-0,459$ & 0,069 & $0,322^{\star \star}$ & $-0,192$ \\
\hline lange Strecken & $0,469^{\star *}$ & 0,359 & 0,462 & 0,220 & 0,013 & 0,130 \\
\hline teures Fahrzeug & $0,310^{\star \star}$ & $-0,119$ & $-0,509$ & 0,020 & 0,081 & 0,037 \\
\hline Fahrkomfort & $0,344^{\star \star}$ & $-0,182$ & $-0,103$ & 0,198 & 0,146 & 0,000 \\
\hline Aktivität: Gespräche & $0,306^{\star \star}$ & 0,078 & 0,250 & 0,174 & 0,212 & $-0,204$ \\
\hline Fahrzeug-Anpassungen & 0,118 & $-0,276$ & $-0,361$ & 0,001 & 0,059 & $-0,114$ \\
\hline kurvige Strecken & $0,382^{\star \star}$ & 0,272 & $-0,365$ & 0,001 & $-0,097$ & 0,222 \\
\hline $\begin{array}{l}\text { ruhig \& vorausschauend } \\
\text { fahren }\end{array}$ & $-0,001$ & $-0,213$ & 0,261 & 0,123 & 0,030 & $0,345^{\star}$ \\
\hline Prestige & $0,308^{* *}$ & 0,261 & $-0,355$ & 0,049 & 0,030 & $-0,331^{*}$ \\
\hline schnell fahren & $0,429^{* *}$ & 0,208 & $-0,642^{\star}$ & 0,077 & $-0,033$ & $-0,104$ \\
\hline gutes Wetter & $0,257^{\star *}$ & $-0,037$ & $-0,053$ & 0,061 & 0,044 & 0,241 \\
\hline produktive Fahrtzeit & 0,063 & 0,140 & $-0,372$ & 0,134 & 0,113 & 0,046 \\
\hline keine Staus/Störungen & 0,176 & 0,184 & $-0,390$ & $-0,001$ & 0,015 & 0,185 \\
\hline keine aggressiven Fahrer & 0,031 & $-0,120$ & $-0,392$ & $-0,131$ & 0,036 & $-0,051$ \\
\hline
\end{tabular}

Anmerkung: Produkt-Moment-Korrelation $r_{x y}$ zwischen Zielvariable „Fahrspaß“ und Faktor; ${ }^{*} p \leq 0,05 ;{ }^{* *} p \leq 0,01$ 